\title{
Berïcksichtigung von Merkmalen der Gesundheit in der Züchtung von Sportpferden
}

\author{
Herrn Professor Dr. Erhard Kallweit zum 65. Geburtstag gewidmet
}

\begin{abstract}
Summary
Title of the paper: Considering health traits in breeding of sport horses

In breeding sport horses performance and health traits are equally important to exhaust the horses' performance potential. The need to consider health traits and to produce sound horses follows also from animal welfare and economic aspects. Often horses not successful in competitions are used in breeding. The time length of active participation in competitions and the reproductive performance of horses are negatively affected by several diseases. Main causes are diseases of locomotor and respiratory organs whereby osteochondrosis dissecans and hemiplegia laryngis are the most frequent ones. These diseases have a sufficiently high heritability and can be selected for. In contrast, reproductive traits in horses have a very low heritability as found in other species. In defining selection policies to improve the health status other factors besides heritability have to be considered such as way of inheritance, frequency of incidence and economic importance. In future the identification of defect genes may help in exactly diagnosing health problems and in breeding for sound constitution. First actions to be taken by breeding organizations relate to testing early progenies. But also actions to improve nutrition, husbandry and training in rearing are important to reduce incidence and relevance of health problems in sport horses.
\end{abstract}

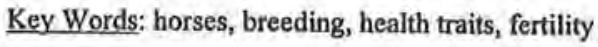

\section{Zusammenfassung}

In der Pferdezucht sind Kriterien der Leistung und Gesundheit gleich wichtig, um das Leistungspotenzial der Pferde ausschőpfen zu können. Die Notwendigkeit der Berlıcksichtigung von Merkmalen der Gesundheit ergibt sich auch aus tierschützerischen und materiellen Grunden. Hăufig werden aber im Turniersport nicht-erfolgreiche Pferde in die Zucht genommen. Die Einsatzdauer der Pferde im Turniersport und deren Fortpflanzung werden durch verschiedene Erkrankungen negativ beeinflusst. Als Hauptursachen sind Erkrankungen der Bewegungsund Atmungsorgane zu nennen, wobei vor allem die Osteochondrose und das Kehlkopfpfeifen hervorzuheben sind. Diese Erkrankungen haben eine ausreichend hohe Heritabilităt und sind somit zuchterisch zu bearbeiten. Im Gegensatz sind Kriterien der Fruchtbarkeit beim Pferd wie bei anderen Tierarten sehr niedrig heritabel. Neben der Heritabilităt sollte bei der Entscheidung uber die Selektion gegen einige Erkrankungen deren Erbgang, Haufigkeit in der Population und deren wirtschafiliche Bedeutung beachtet werden. Zukunftig kann die Identifizierung von Defektgenen die exakte Diagnose und zlichterische Berlicksichtigung erleichtern. Als erste züchterische Massnahmen werden den Zuchtorganisationen Prüfungen von Nachkommen aus den ersten Deckjahren der Hengste empfohlen. Aber auch Massnahmen zur Verbesserung der Ernahrung, Haltung und des Trainings wăhrend der Aufzucht sind erforderlich, um die Hăufigkeit von Erkrankungen zu reduzieren.

Schlusselworter: Pferde, Zucht, Gesundheit, Fruchtbarkeit

1. Einleitung

In der Pferdezucht, ob bei Reit-, Renn- oder Freizeitpferden, sind Kriterien der Leistung und Konstitution gleich wichtig. Auf die Bedeutung gesundheitlicher Aspekte in 
der Pferdezucht im Vergleich zu anderen Tierarten hat GLODEK (1979) hingewiesen. Während die Selektion auf Einzelmerkmale, wie z.B. auf Muskelwachstum beim Schwein und Huhn, zu Skelettschäden geführt hat, werden derartige Nebeneffekte in der Pferdezucht als eher unwahrscheinlich betrachtet. Unabhängig von der Nutzungsart der Pferde sind im allgemeinen grosse Investitionen getätigt worden, bevor das Pferd seiner eigentlichen Nutzung zugeführt werden kann. Deshalb ist es auch aus materiellen Gründen notwendig, Pferde zu züchten, die von ihrer Konstitution her gesund sind, um ihr Leistungspotenzial ausschöpfen zu können. Nicht zuletzt verlangt der Tierschutz, dass Aspekte der Gesundheit in der Pferdezucht berücksichtigt werden. Zwar werden in der Pferdezucht durch die genetische Verbesserung z.B. der Rittigkeit, der Springveranlagung und der Schnelligkeit unerwünschte Nebeneffekte selten sein; doch birgt das System der Leistungsprüfung und Selektion von jungen Pferden Gefahren in sich, da manche konstitutionellen Mängel erst nach der Selektion und in höherem Alter überprüft werden können. Hinzukommt ein züchterisches Fehlverhalten, dass im Sport nicht-erfolgreiche Pferde bzw. Pferde nach operativen Eingriffen in die Zucht genommen werden. Deshalb sollen anhand von eigenen Untersuchungen und von Literaturergebnissen Möglichkeiten aufgezeigt werden, wie bei der Zucht von leistungsveranlagten Pferden Aspekte der Gesundheit stărker als bisher beachtet werden können.

\section{Bedeutende Erkrankungen und deren Ursachen}

In der Zucht von Sportpferden spielen Kriterien wie das Exterieur, die Rittigkeit und die Springveranlagung die grösste Rolle. Diese Kriterien zeigen gerade in der subjektiven Bewertung durch mehrere Richter / Reiter bei einer stationären Prüfung der Hengste oder Stuten eine mittelgrosse Heritabilität zwischen $30 \%$ und $50 \%$ und lassen ausreichend grosse Zuchtfortschritte nach Selektion erwarten. Hinsichtlich der Gesundheitsaspekte gibt es eine Reihe von verschiedenen Erkrankungen, die die Einsatzdauer der Pferde im Sport und ihre Fortpflanzung negativ beeinflussen können; eine aktuelle Zusammenstellung findet sich bei NICHOLAS (2000) und COLLINDER und RASMUSON (2000). Die Erkrankungen sind hinsichtlich ihrer Häufigkeit und relativen Bedeutung zu werten (Tab. 1). Als Hauptursachen erweisen sich in mehreren Untersuchungen Mängel im Bewegungs-, Atmungs-, Herz- und Kreislauf und Verdauungsapparat, wobei die Ergebnisse stark vom verfuggaren Datenmaterial abhängen. Aufgrund von deutschen Erhebungen aus Versicherungsstatistiken und Untersuchun-

Tabelle I

Hăufigkeit der Ausfallursachen von Pferden (Frequencies of diseases in horses)

\begin{tabular}{lcccc}
\hline Erkrankung der ..-organe & \multicolumn{3}{c}{$\begin{array}{c}\text { Häufigkeit erkrankter Pferde (\%) } \\
\text { LINDNER \& } \\
\text { HOMMERICH } \\
(1995)^{*}\end{array}$} & $\begin{array}{c}\text { WALLIN et al. } \\
(2000 \mathrm{~b})\end{array}$ \\
\hline Bewegungs- & 61.2 & 40.5 & 51.4 & 60.1 \\
Verdauungs- & 9,8 & 9,0 & 14,4 & 5,6 \\
Herz-/Kreislauf- & 4,6 & & 8,1 & 2,6 \\
Harn-/Geschlechts-/andere & 1,2 & & 5,2 & 4,1 \\
Atmungs- & 16,3 & 16,5 & 12,2 & 8,9 \\
Infektionen $/$ Nerven- & 2,8 & & 2,3 & 5,4 \\
\hline
\end{tabular}

"Durchschnitt der Jahre 1984-1994, "0 Durchschnitt aus 1971-1984 
gen aus Schweden sind bei Warmblutpferden Störungen des Bewegungsapparates mit ca. $56 \%$ und Erkrankungen der Atemwege mit etwa 11\% die häufigsten Ausfallursachen. Störungen im Verdauungstrakt treten mit einer Frequenz von unter $10 \%$ auf und Erkrankungen des Nervensystems sind mit ca. $3 \%$ eher unbedeutend.

Die genetischen Ursachen einzelner weniger Erkrankungen sind mit Hilfe der Molekulargenetik geklärt. Dabei kann man unterscheiden zwischen Erkrankungen, die von einzelnen oder mehreren Genen beeinflusst werden (vgl. COLLINDER und RASMUSON, 2000). Die sog. Erbdefekte scheinen beim Pferd, vor allem in der Warmblutzucht, insgesamt selten vorzukommen. So z.B. die hyperkaliämische periodische Paralyse (HYPP) beim Quarter Horse, eine Muskelerkrankung, die autosomal dominant vererbt wird und für die ein Gentest inzwischen vorliegt (BOWLING et al., 1996); die schwere kombinierte Immundefizienz (SCID) beim Araber, die autosomal recessiv vererbt wird und mittels eines diagnostischen DNA-Testes identifiziert werden kann (BERNOCO und BAILEY, 1998); andere erwähnenswerte Defekte sind die weissgeborenen Fohlen mit dem sog. Lethal White Foal Syndrom (LWFS) bei den Paint- und Pinto-Pferden und Anomalien der Gliedmassen bei Shetlandponies (PHILIPSSON et al., 1998; HERMANS et al., 1987). Für andere Erkrankungen, z.B. für die chronische Bronchitis, Kryptorchismus und Osteochondrosis, geht man von multifaktoriellen Ursachen aus; niedrige Heritabilitäten im Bereich von ca. 0,20, Einfluss von Aufzucht und Haltung sowie Wechselwirkungen von genetischen und umweltbedingten Effekten, eventuell auch mit der Selektion auf Springvermögen und Rittigkeit korrelierte Effekte werden als mögliche Einflussgrössen angesehen. Im folgenden soll auf einige Erkrankungen beispielhaft eingegangen werden, um züchterische Möglichkeiten zur Reduktion ihres Vorkommens abzuleiten.

\subsection{Gliedmassenerkrankungen}

Aufgrund der hohen Verluste in der Sportpferdezucht sind in den letzten Jahren verschiedene Untersuchungen in den Niederlanden, Schweden und Deutschland durchgeführt worden, um die Häufigkeit und Erblichkeit verschiedener Gliedmassenerkrankungen festzustellen. Dabei wurden nur solche Erkrankungen einbezogen, die röntgenologisch zu bewerten waren. Die Ergebnisse schwanken sehr stark zwischen den Untersuchungen bedingt u.a. durch grosse Unterschiede im Tiermaterial, das von ausgewählten Auktionspferden bis zu nicht-selektierten Hengstnachkommenschaften reicht (Tab. 2). Dies spiegelt sich in den unterschiedlichen Frequenzen und den Schätzungen der Heritabilität wider. Trotz aller Unterschiede muss man zu dem Schluss kommen, dass bei den untersuchten Erkrankungen des Bewegungsapparates röntgenologische Befunde eine Bewertung erlauben.

Die Osteochondrose (Osteochondrosis dissecans, OCD) ist eine der am häufigsten vorkommende Gliedmassenerkrankung bei wachsenden Pferden. Schon im Fohlenalter soll nach KROLL (2000) Osteochondrose röntgenologisch nachweisbar sein. Rassenunterschiede in der Häufigkeit sind allgemein akzeptiert. Ponys haben selten Osteochondrose, bei Trabern und Reitpferden liegt die Frequenz von Osteochondrose wohl bei 10 bis $15 \%$. Die Schätzungen der Heritabilität schwanken je nach der angewandten Methodik und der untersuchten Population zwischen 0,1 und 0,3 (Tab. 2). 
Tabelle 2

Hăufigkeiten von Veränderungen an den Gliedmassen von Warmblutpferden und deren Heritabilitătsschătzwerte (Frequencies of bone lesions in warmblood horses and their estimates of heritability)

\begin{tabular}{lccccccc}
\hline Erkrankung & \multicolumn{3}{c}{ Keine Abweichungen von der } & \multicolumn{3}{c}{ Heritabilitat } \\
& G & Norm (\%) & K & N & G & K & N \\
\hline Hufrollenentzlandung & 21 & 23 & 27 & 0,06 & 0,31 & 0,30 \\
Arthrose der Zehengelenke & 63 & 34 & 89 & 0,05 & 0,29 & 0,23 \\
Hufknorpelverknocherung & 79 & 14 & & 0,18 & 0,32 & \\
Gleichbeinlahmheit & 87 & 64 & 39 & 0,16 & 0,34 & 0,13 \\
Osteochondrosis dissecans & 89 & 95 & 86 & 0,07 & 0,34 & 0,05 \\
Spat & 7 & 81 & 85 & 0,04 & 0,35 & 0,27 \\
\hline
\end{tabular}

Daten: G 3566 Auktionspferde, 862 Vater (WINTER, 1995)

K 456 Stuten, 97 Vater (WILLMS, 1999)

N 600 Stuten, 30 Vater (KWPN, 1994)

Hinsichtlich der Osteochondrose, die in einigen Zuchtpopulationen eine besondere Beachtung erfährt, sind weitere Erkenntnisse von Bedeutung:

In der Reitpferdezucht zeigt sich eine negative Beziehung zur Rittigkeit und Springveranlagung, d.h. Osteochondrose wird häufiger festgestellt bei überdurchschnittlichen Dressur- und Springpferden (WINTER, 1995).

In der Rennpferdezucht haben die Pferde mit mehrfachen Osteochondrose-Befunden weniger Starts und einen schlechteren Leistungsindex (PHILIPSSON, 1999)

In deutschen Untersuchungen konnte KROLL (2000) zeigen, dass ein OCD-Befund sich beim Fohlen bis zum sechsten Lebensmonat bereits manifestiert hat. Untersuchungen aus den Niederlanden deuten an, dass OCD-Befunde des Fohlens im späteren Alter wieder verschwinden können bzw. Fohlen, die OCD frei sind, im späteren Alter OCD bekommen können (BARNEVELD et al., 1999).

Die Ursachen von OCD sind multifaktoriell, d.h. die Ernăhrung der Mutterstute und des Fohlens, die Bewegungsart und -hăufigkeit sind bedeutende umweltbedingte Einflussfaktoren neben der genetischen Veranlagung. Auf die positiven Effekte durch regelmässige Bewegung von Fohlen, am besten in Gruppenhaltung auf der Weide, weisen HERTSCH und KROLL (1999) und BARNEVELD et al. (1999) hin.

Erste Erkenntnisse zur molekulargenetischen Diagnostik der OCD sind von DE BACKER (2000) vorgestellt worden.

\section{$2.2 \quad$ Atemwegserkrankung}

Hier sei nur auf das sog. Kehlkopfpfeifen (Hemiplegia laryngis) eingegangen, eine weitverbreitete und seit langem bekannte Erkrankung des Kehlkopfes, die als Hauptmangel in der Pferdezucht seit Ende des 19. Jahrhunderts bekannt ist, deren Ätiologie aber nicht gänzlich geklärt ist. Letztlich fuhrt ein atrophierter Muskel bei Belastung der Pferde zu Atemgeräuschen und -beschwerden. In einer umfangreichen Studie an ca. 1000 Pferden aus der deutschen Warmblutzucht hat MIESNER (1996) einen Indexwert für die linksseitige abduktorische Kehlkopffunktionsstörung abgeleitet zur Einteilung der untersuchten Pferde in Befundklassen; danach zeigten ca. $25 \%$ der untersuchten Fohlen und ca. 50 bis $60 \%$ der untersuchten älteren Pferde deutliche Befunde. Die Schätzwerte der Heritabilität liegen mit einem Wert von 0,60 sehr hoch und weisen auf 
gute Möglichkeiten der Selektion gegen diese Atemwegserkrankung hin. Auch zeigte sich in der Untersuchung deutlich, dass Hengste und Stuten als Elterntiere mit deutlichen Befunden mehr Nachkommen mit Kehlkopfpfeifen erzeugen als Elterntiere ohne Befunde. Andere Faktoren wie z.B. Infektionen können aber auch zum Auftreten der Erkrankung beitragen. Prädisponiert für diese Erkrankung scheinen grosse Pferde mit leichter Halsung zu sein. MIESNER (1996) empfiehlt aufgrund der genetischen Parameter, in die Zuchtprogramme der Reitpferdezucht die Selektion gegen diese Atemwegserkrankung aufzunehmen.

\section{$2.3 \quad$ Einsatzdauer im Turniersport}

Alle Erkrankungen der Sportpferde führen letztlich zu einer verkürzten bzw. abgebrochenen Karriere im Sport. Die Einsatzdauer im Sport ist deshalb ein indirekter Indikator für die Gesundheit der Sportpferde. In eigenen Untersuchungen an Turnierpferden wurde als Hilfsparameter für die Einsatzdauer die Zeitdauer zwischen der ersten und letzten Platzierung eines Pferdes auf den A/B-Turnieren gewählt (WÖHLK und BRUNS, 1999). Da auch ohne eine Platzierung ein Pferd noch im Turniersport eingesetzt werden kann, erscheint die oben definierte Einsatzdauer eher eine Unterschätzung der wahren Einsatzdauer zu sein. Anhand dieses Parameters zeigte sich, dass $50 \%$ aller Turnierpferde nicht länger als zwei Jahre im Sport verbleiben; Hengste und Wallache sind im Durchschnitt etwa 3,5 Jahre und damit 0,5 Jahre länger als Stuten im Sport. Hingegen schätzt GUTEKUNST (1977) anhand von Versicherungsstatistiken die mittlere Einsatzdauer von Sportpferden im Turniersport auf etwa 5,5 Jahre. Pferde, die ihre Sportkarriere mit vier bis funf Jahren beginnen, erreichen über fast vier Jahre Platzierungen im Turniersport, während bei Beginn neun- bis zehnjährige Pferde nur etwas mehr als zwei Jahre erfolgreich sind. Erfreulich ist auch, dass Turnierpferde, deren Väter einen überdurchschnittlich hohen Leistungsindex (über 120 Punkte) haben, mindestens vier Jahre lang Platzierungen erreichen, während Pferde von unterdurchschnittlichen Vätern weniger als drei Jahre lang erfolgreich sind.

Diese Ergebnisse deuten auf eine relativ kurze Nutzung der Pferde im Sport hin, lediglich die besonders erfolgreichen Pferde und solche mit erfolgversprechender Abstammung verbleiben länger im Sport. Letzteres ist züchterisch erwünscht und unterstreicht gleichzeitig die Richtigkeit der Leistungsbewertung der Hengste anhand der Turniersportleistung ihrer Nachkommen. Andererseits verschweigen die Ergebnisse die wahren Gründe, warum Pferde nicht länger im Turniersport erfolgreich sind bzw. ganz aus dem Sport ausscheiden. Eine ähnlich unzureichende Datengrundlage über wahre Ausscheidungsgründe bemängeln auch LINDNER und OFFENEY (1992) sowohl für Renn- als auch Reitpferde. Deshalb wäre es wünschenswert, wenn die Zucht- und Sportorganisationen zukünftig Informationen über die wahren Ausscheidungsgründe der Pferde erfassen könnten. In einer Survival-Analyse anhand von schwedischen Reitpferden zeigen WALLIN et al. (2000) die Bedeutung von korrekten und gesunden Gliedmassen der Sportpferde für deren Langlebigkeit; auch zeigt sich, dass Pferde mit einer schlechten Benotung in der Spring- und Dressurveranlagung eine kürzere Lebensdauer haben als Pferde mit einer besseren Benotung.

Einen positiven Effekt auf eine lange Einsatzdauer im Sport kann die Gruppenhaltung 
von Pferden ausüben. Während SCHÄFER et al. (2000) auf den Trainingseffekt und die verbesserte Fitness der in Gruppen gehaltenen Pferde hinweisen, deuten die Untersuchungen von SONDERGAARD und SCHOUGAARD (2000) auf eine grössere Langlebigkeit der Pferde hin, wenn die Pferde statt in Einzelboxen in Gruppen mit Auslauf gehalten werden.

\section{$2.4 \quad$ Fruchtbarkeit}

In der gesamten Nutztierzucht hat die Fruchtbarkeit eine besonders hohe wirtschaftiche Bedeutung. In der Pferdezucht sind die Kosten für die Erzeugung eines Fohlens im Vergleich zu anderen Nutztierarten sehr hoch. Deshalb sind alle Kriterien, die die Lebensfähigkeit eines Fohlens, vom Embryo bis zum Neugeborenen, beeinflussen, von besonderem Interesse. Es ist also wichtig, dass möglichst viele der gedeckten Stuten tragend werden und dass diese Stuten ein gesundes Fohlen zur Welt bringen.

In unserer jüngsten Studie an Daten aus dem hannoverschen Zuchtgebiet konnten signifikante Einflüsse des Deckjahres und der Befruchtungsart sowie des Alters von Stuten und Hengsten festgestellt werden (DOHMS und BRUNS, 2000). So schwankt die mittlere Befruchtungsrate zwischen 71\% im Jahre 1996 bis 78\% im Jahre 1992. Ebenso deutlich unterscheiden sich die Deckarten in der Befruchtungsrate, bei Hofbesamung, d.h. bei Frischbesamung im Züchterstall, wird nur eine Befruchtungsrate von $72 \%$ erreicht, während bei Natursprung und Frischsamenübertragung auf Deckstationen eine Befruchtungsrate von $76 \%$ erreicht wird. Die in Tabelle 3 dargestellten Ergebnisse der Varianzkomponentenschätzung zeigen, dass für die Merkmale der Stutenfruchtbarkeit die Heritabilitäten sehr niedrig sind, wie auch in anderen Studien anhand von Daten der Holsteiner Zucht (NISSEN, 1986; WILKENS, 1989) und bei anderen Nutztierarten gezeigt.

Tabelle 3

Schätzwerte von Varianzkomponenten für die Merkmale Befruchtungsrate, Gulstzeit und Zwischenfohlzeit (Estimates of variance componenets of pregnancy rate, days open, foaling interval)

\begin{tabular}{lccc}
\hline Varianzursache & \multicolumn{3}{c}{ Varianzkomponenten (relativ) } \\
& Befruchtungsrate & Gustzeit & Zwischenfohlzeit \\
\hline Additiv genetisch & 0,02 & 0,01 & 0,01 \\
Permanent umweltbedingt & 0,03 & 0,00 & 0,00 \\
Deckhengst & 0,01 & 0,01 & 0,01 \\
Deckstation & 0,04 & 0,00 & 0,01 \\
Restabweichung & 0,90 & 0,98 & 0,97 \\
\hline
\end{tabular}

Datenmaterial: 38850 Stuten des hannoverschen Zuchtverbandes

Bei der Stutenfruchtbarkeit liegt eine Problematik in der Genauigkeit und Standardisierung der Datenerfassung. Solange nicht jede Bedeckung, unabhängig von der Bedeckungsart, sofort der Zuchtorganisation mitgeteilt wird, werden keine genauen und einheitlich erfassten Fruchtbarkeitsparameter für alle Hengste einer Population zur Verfügung stehen. Für Hengste im Privat- und Staatsbesitz, für Hengste, die im Naturspung decken oder in der künstlichen Besamung eingesétzt werden, müssen einheitliche Bewertungskriterien gelten. Ergebnisse der regelmässigen Spermauntersuchungen und Angaben zur Non-Return-Rate (70 Tage) sollten den Stutenbesitzern zu Beginn der Decksaison mitgeteilt werden, damit diese bei der Hengstauswahl sowohl Kri- 
terien der Leistung (Rittigkeit, Springen) als auch Kriterien der Fruchtbarkeit gleichzeitig und abwägend berücksichtigen können. Für die Verbesserung der Fruchtbarkeit sollten aber nicht nur züchterische Möglichkeiten genutzt werden, sondern die Stutenbesitzer sind seitens der Zuchtorganisationen auch über die Möglichkeiten und Wirkungen von Managementmassnahmen, wie z.B. Zuchthygienemassnahmen und Impfprogrammen, zu informieren.

\section{Gesundheitsaspekte in der Züchtung}

Die Beachtung von Gesundheitsaspekten in der Züchtung von Sportpferden ist eine häufig gestellte Forderung, da das allgemeine Zuchtziel Kriterien der Leistung und Gesundheit gleichgewichtig betrachtet. Dies bedeutet aber nicht, dass jede Erkrankung züchterisch berücksichtigt werden muss. Man sollte sich vergegenwärtigen, dass je mehr Merkmale gleichzeitig züchterisch zu verbessern sind, um so geringer ist der Erfolg in jedem einzelnen Merkmal, also sowohl in den Leistungs- als auch in den Gesundheitsparametern. Für die Abwägung, welche Erkrankungen neben den Kriterien der Leistung züchterisch zu verbessern sind, sollte die Häufigkeit des Auftretens der Erkrankung, deren wirtschaftliche Bedeutung und deren Erbgang und Erblichkeitsgrad beachtet werden. Die Beziehung der Erkrankung zu den wichtigen Leistungseigenschaften sollte ebenso für die Entscheidung herangezogen werden. Eine exakte Diagnose und routinemässige populationsweite Erfassung der Erkrankung über mehrere Jahre ist erforderlich, bevor eine züchterische Entscheidung getroffen werden kann. Bei einzelnen Krankheiten könnte die Identifizierung von Defektgenen in Zukunft die exakte Diagnose und die züchterische Berücksichtigung erleichtern, siehe HYPP, LWFS oder vielleicht in Zukunft auch OCD.

Was kann der Einzelzüchter bzw, die Zuchtorganisation tun? Konkret können folgende Massnahmen kurzfristig in die Praxis umgesetzt werden, wenn man z.B. die Situation mit der Osteochondrose bzw. dem Kehlkopfpfeifen verbessern will:

- Der Gesundheitsstatus aller potenziellen Vatertiere sollte zum Zeitpunkt der Körung erfasst werden. Die Nachkommen aus den ersten Deckjahren (ca. 20 bis 30 Nachkommen) sollten einer vollständigen Kontrolle, z.B. im Fohlenalter oder bis spätestens zum Zeitpunkt der Stutbuchaufnahme im Alter von zwei bis drei Jahren, unterzogen werden.

- Die schlechtesten Zuchttiere soliten aus der Zucht herausgenommen werden; dies könnten Hengste sein, deren Osteochondrose-Befund mit drei bis vier bewertet wurde; auch sollten operierte Pferde nicht zur Zucht zugelassen werden, ev. Ausnahmen sind sorgfältig zu prüfen.

- Eine Beschränkung auf wenige, wichtige Erkrankungen ist notwendig.

- Ein Selektionsprogramm, das unterdurchschnittliche Kandidaten sowohl in Kriterien der Leistung als auch der Gesundheit nicht zur Zucht zulässt, ist strikt umzusetzen.

- Eine ausführliche Dokumentation des züchterischen Wertes von Zuchttieren, vor allem von Hengsten, ist zwingend erforderlich und schliesst Kriterien der Reiteignung, Fruchtbarkeit und Gesundheit ein, auch aus Gründen der Produkthaftung.

Was sollte aber neben der Züchtung noch in der Erzeugung von Sportpferden beachtet 
werden? Bei den meisten Erkrankungen erklärt die Genetik nur den kleineren Teil der Ursachen. Häufig verursachen Mängel in der Ernährung, Haltung, Aufzucht und dem Training der Pferde das Auftreten der Erkrankungen.

- Vor allem sollten die Ernährung und Haltung während der Aufzucht, d,h. vom Fohlen bei der Mutter bis hin zum Dreijährigen, optimiert werden. Eine Überfütterung mit verdaulicher Energie und Protein ist zu vermeiden, die Aufzuchtintensität sollte reduziert werden und Imbalanzen bei den Mineralstoffen sind zu vermeiden, Dies setzt exakte Analysen des Grund- und Leistungsfutters voraus.

- Die Haltung und das Training der Reitpferde während der Ausbildung sind hinsichtlich Intensität und Dauer zu optimieren. Haltungsformen, die dauernde und grosszügige Bewegungsmöglichkeiten schon in der Fohlenaufzucht eröffnen, sind ideal.

Für die Zucht von leistungsfähigen, gesunden und fruchtbaren Sportpferden sind letztlich Erkenntnisse der Genetik und Züchtung, der Haltung und Ernährung, sowie der Veterinärmedizin zusammenzuführen. Die Zuchtorganisationen sollten neben der eigentlichen Züchtungsarbeit auch beratend in der Haltung und Ernährung tätig werden. Eine enge Zusammenarbeit mit der Veterinärmedizin ist angezeigt zur Entwicklung und Durchführung von Hygiene- und Impfprogrammen.

\section{Literatur}

BARNEVELD, A.; VAN WEEREN, R.; KNAAP, J:

Influence of early exercise on the locomotion system. Proc. 50th Annual Meeting of the European Association for Animal Production (EAAP), August 23-26, Zurich, Switzerland, 1999

BERNOCO, D.; BAILEY, E.: Frequency of the SCID gene among Arabian horses in the USA. Animal Genetics 29 (1998), $41-42$

BOWLING, A.T.; BYRNS, G.; SPIER, S.:

Evidence for a single pedigree source of the hyperkalemic periodic paralysis susceptibility gene in Quarter Horses. Animal Genetics 27 (1996), 279-281

CLAUSEN, M.; PREISINGER, R.; KALM, E.:

Analyse von Krankheitsdaten in der deutschen Warmblutzucht. Zuchtungskunde 62 (1990), 167-178

COLLINDER, E.; RASMUSON, M.: Genetic aspects of disease in horses. The Genetics of the Horse. CAB Int'l. (2000), 157-170

DE BACKER, L.: Van veulen tot sportpaard. Vortag Universittat Utrecht „Symposium 2000", IS. Dezember 2000

DOHMS, T,; BRUNS, E.: Einfluss von umweltbedingten und genetischen Faktoren auf die Fruchtbarkeit von Stuten. Vortragstagung der GDFZ/GFT, 21,122. September 2000, Kiel

GLODEK, P.: Merkmalsantagonismen in der Leistungszucht bei Pferden. Zuchtungskunde 51 (1979), 459-467

GUTEKUNST, H.P.:

Zur Schadensursachenstatistik von entschadigten Reitpferden in den Jahren 1971-1974 innerhalb der BRD einschliesslich Westberlin. Uniy. Giessen, Diss, 1977

HERMANS, W.A.; KERSJES, A.W,; VAN DER MEY, G.J.; DIK, K.J.: Investigation into the heredity of congenital lateral patellar (sub)luxation in the Shetland pony. Veterinary Quarterly 9, 1-8 (1987)

HERTSCH, B; KROLL, A.: Skelettreife beim Fohlen. G8ttinger Pferdetage '99 (1999), 215-222

HOMMERICH, G.: Ausfallursachen und-frequenzen. Gottinger Pferdetage '95 (1995), 85-94 KROLL, A.: Zur Osteochondrosis dissecans beim Saugfohlen. Freie Universitat Berlin, Diss, 2000 
KWPN:

The frequency and heredity of navicular disease, sesamoidosis, fetlock joint arthrosis, bone spavin, osteochondrosis of the hock. A radiographic progeny study. KWPN, Zeist (1994)

LINDNER, A.; OFFENEY, F: MIESNER, K :

Einsatzdauer, Abgangsraten und -ursachen bei Sportpferden. Dtsch. Tierărztl. Wsch. 99 (1992) 1, 39-42 Untersuchungen uber die abduktorische Funktionsstorung des Kehlkopfes beim Pferd-Genetische und umweltbedingte Einflussfaktoren sowie mogliche zllchterische Massnahmen. Rheinische FriedrichWilhelms-Universitatt Bonn, Diss., 1996

NICHOLAS, F.W:

Genetics of morphological traits and inherited disorders. The Genetics of the Horse. CAB Int'1. (2000), NISSEN, T.: 71-84

Stutenprufung beim Holsteiner Warmblutpferd - Leistungsveranlagung und Fruchtbarkeit. ChristianAlbrechts-Universitat Kiel, Diss., 1986

PHILIPSSON, J.; BRENDOV, E.; DALIN, G.; WALLIN, L.:

Genetic aspects of diseases and lesions in horses. World Conference on Genetics applied to Livestock Production, Armidale, Australia (1998) 24, 408-415

PHILIPSSON, J.:

Genetische Aspekte von Erkrankungen beim Pferd. Gottinger Pferdetage '99 (1999), 11-22

SCHÄFER, B; ELLENDORFF, F.; KALLWEIT, E.:

Influence of housing and detraining on fitness of sport horses. Proc. $51^{\text {st }}$ Anmual Meeting of the European Association for Animal Production (EAAP), August 21-24, The Hague, Netherlands, 2000

WALLIN, L; STRANDBERG, E.; PHILIPSSON, J.:

Longevity of Swedish warmblood horses and phenotypic relationships with their test results as four-yearolds. Proc. $51^{\text {st }}$ Annual Meeting of the European Association for Animal Production (EAAP), August 2124, The Hague, Netherlands, 2000a

WALLIN, L.; STRANDBERG, E.; PHILIPSSON, J.; DALIN,G.:

Estimates of longevity and causes of culling and death in Swedish warmblood and coldblood horses. Livest. Prod. Sci, 63 (2000b) 3, 275-289

WILKENS, J.:

Analyse der Fruchtbarkeit und der Inzucht beim HoIsteiner Warmblutpferd. Christian-AlbrechtsWILLMS, F. Universitat Kiel, Diss., 1989

Genetische Analyse von Merkmalskomplexen der Reitpferdezucht unter Berucksichtigung von WINTER, D.: Gliedmassenverănderungen, Christian-Albrechts-Universitatt Kiel, Diss., 1998

Genetische Disposition von Gliedmassenerkrankungen bei Reitpferden. Georg-August-Universität Göttingen, Diss., 1995

WÖHLK, K.; BRUNS, E:

Analyse der Nutzungsdauer von Reitpferden im Turniersport. Gottinger Pferdetage '99 (1999), 31-40

Eingegangen: 16.01 .2001

Akzepticrt: 09.02 .2001

Anschrift des Verfassers

Prof. Dr. ERICH BRUNS

Institut fur Tierzucht und Haustiergenetik

Georg-August-Universităt Göttingen

Albrecht-Thaer-Weg 3

D-37075 Gottingen

E-Mail: ebruns@gwdg.de 


\title{
Buchbesprechung
}

Beiträge zur Jagd- und Wildforschung, 22, und 23. Band

\author{
M. STUBBE (Hrsg.) \\ Im Auftrag der Gesellschaft fur Wildtier- und Jagdforschung
}

Bd. 22, 398 Seiten, Bd. 23, 320 Seiten, zahlreiche Tabellen und Abbildungen, Burghausen, 1997, 1998

$\mathrm{Zu}$ beziehen durch: Geschätsstelle der Gesellschaft filr Wildtier- und Jagdforschung e.V., Schkeuditzerstr. 33, D-04430 Burghausen, je Band DM 38,00 + Versandkosten

Beide Bănde dieser Schriftenreihe dokumentieren neueste Ergebnisse aus allen Bereichen der Wildtier- und Jagdforschung. Wie in den vorangegangenen Bänden, werden aktuelle Erkenntnisse zur Biologie, Ökologie, zum Management und Schutz bewirtschafteter und bedrohter Săugetiere und Vogelarten vermittelt. In einem weiten Spektrum enthalten diese Bucher Beiträge zum Arten- und Biotop- sowie Umweltschutz, ebenso wie Beiträge uber die Bestrebungen ordnungsgemaße Wildbewirtschaftung, Waldwirtschaft und Agrarlandnutzung in Einklang zu bringen. Die nationalen und internationalen, auf der Grundlage fundierter Forschungsergebnisse, vorgestellten Beiträge verdeutlichen einmal mehr, dass es im modernen okologischen Verstăndnis zwischen Jagd und Naturschutz, zwischen Ressourcenschutz und Ressourcennutzung keine unløsbaren Konflikte gibt.

An den Einfuhrungsbeitrag im Bd. 22 uber Naturressourcen der offenen Landschaft und ihrer Perspektiven schließt sich das Thema uber die Bedeutung von Feuchtgebieten in Natur und Gesellschaft an. Es folgen Beiträge uber die Bestände der Saat- und Blaßgans sowie Schäden durch Wildgănse auf Agrarflăchen in der Tschechischen Republik. Eine grßßere Anzahl von Beiträgen beschäftigen sich mit den Feldhasen, seinen Bestanden in verschiedenen Gebieten Deutschlands, Methoden der Bestandsschätzung, Einflussen auf die Bestandsentwicklung, der Tiergesundheit, der Bestandsforderung bis zu molekulargenetischen Untersuchungen an Feldhasenbeständen Hessens. Eine ăhinliche Themenvielfalt ist dem Rotfuchs gewidmet. Es folgen Beiträge mit Untersuchungen an Mauswiesel, Haselhuhn und Rauchfußhühnern sowie eine größere Anzahl Arbeiten uber Rotwild, Mufflon, Damwild und dem Rehwild mehrerer Standorte, untersucht von Mecklenburg-Vorpommern bis zum Allgäu. Sie beinhalten u.a. Fragen territorialer Ausbreitung, des Wanderungs - bzw, Raum-Zeitverhaltens, der Bestandsregulierung, von Fallwildanteilen, der Gesundheit, bestimmter Futterungseinflusse und der Trophäen. Die letzten beiden der 38 Beiträge beschäftigen sich mit der Wiederansiedlung des Luchses im Harz und den Wildeinbürgerungen in der Schorfheide.

Der Bd. 23 enthalt einen Einfuhrungsbeitrag zum Schutz von Naturressourcen an der Schwelle des 21 . Jahrhunderss. Die meisten der 34 Beitrăge beschaftigen sich mit wildøkologischer Lebensraumbewertung, Bestandsbewertungen, Wildeinbllrgerungen, Trophäenentwicklungen, Raumverhalten, der Hege oder Tierschutz und Jagd von Wild in verschiedenen Gebieten, oberwiegend Deutschlands. Zu den beschriebenen Wildarten zăhlen u.a. Rotwild, Damhirsch, Muffel- und Gemswild, Braunbar, Baum- und Steinmarder, Fischotter, Dachs, Luchs sowie verschicdene Raub- und Wasservogel.

Den Vakatseiten dieser Bucher sind u.a. Tagungsankundigungen, Rezensionen, Buchangebote und Mitteilungen der Gesellschaft für Wildtier- und Jagdforschung zu entnehmen.

Diese interessanten, breitgefacherten, lesenswerten Blucher enthalten sowohl wichtige Basis- und Hintergrundinformationen für eine waidgerechte Jagd und wissenschaftlich begrindete Wildbewirtschaftung als auch aktuelle Informationen für den Schutz und den Nutzen von Wildressourcen. Neben Biologen, Zoologen, Ökologen, breiten Kreisen der Jägerschaft, Land- und Forstwirten, Tierärzten sind sie land- und forstwirtschaftlich ausgerichteten Institutionen sowie allen Naturfreunden zu empfehlen. 